\title{
Barton F. Branstetter 4th, Daniel L. Rubin, D. Scott Griffin, David L. Weiss (eds): Practical Imaging Informatics. Foundations and Applications for PACS Professionals
}

\author{
Springer New York Dordrecht Heidelberg London, ISBN 978-1-4419-0483-6 e-ISBN 978- \\ 1-4419-0485-0, http://www.springer.com/978-1-4419-0483-6, (C) 2009 Society for Imaging \\ Informatics in Medicine (SIIM), published in 2010
}

\section{Thomas M. Deserno}

Published online: 4 November 2011

(C) Society for Imaging Informatics in Medicine 2011

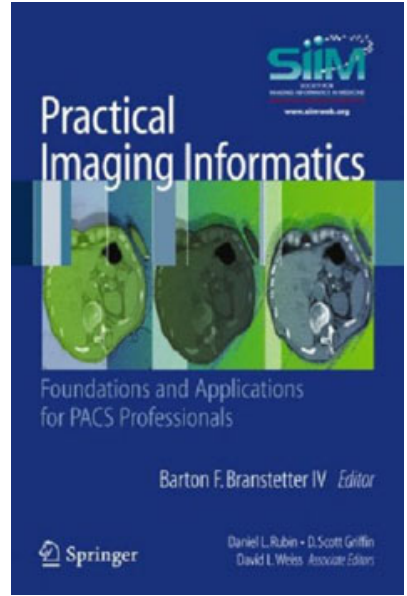

\section{Book Review}

Medical informatics has been established as a self-standing discipline bridging computer science and medicine. Since information systems have become essential part of today's health care, the need of qualified personnel for development, installing, and operating such systems has been manifested, and such specialists need profound knowledge in both clinical workflow and computer technologies. In particular, this holds for departments of radiology, which

\section{T. M. Deserno $(\triangle)$}

Department of Medical Informatics, RWTH Aachen University, Pauwelsstraße 30,

52074 Aachen, Germany

e-mail: deserno@ieee.org have become fully digital interconnecting computer systems for image acquisition, reading, decision making, and intervention. Here, imaging informatics professionals (IIP) are required living in both, the medical and information technology (IT) culture, and this book is written to support their daily work. Furthermore, IT staff, physicians, trainees, and healthcare administrators are targeted by the book that presents a suitable reference and troubleshooting guide for everyday imaging informatics. So, practical and useful application of modern IT in the healthcare is clearly focused rather than scientific disputes and proofs.

The book is organized in three sections (Technology, Operation, and Strategy and Vision), each of which having assigned its own associated editor, and each of which is being organized in two parts consisting of three to five individual chapters, a rather fundamental and another more practical part. Most of the chapters, in total 26, are written as a monograph of about 15 pages, and the respective author has been selected carefully from leading experts in the field. These authors form a good mixture of academics and research, technicians and technical managers, as well as consultants and project managers, and all are associated within the United States. A comprehensive glossary and a detailed index are completing this outstanding resource of knowledge.

All chapters follow an equal structure and type of writing, which let the entire compilation appear as a monograph rather than an edited work. The chapters are framed by a brief introduction, and suggested reading as well as a collection of multiple choice questions for self-assessment; the answer key is also given in the book. Each section/subsection is further structured by bullet points, offering the readers to rapidly access the required item of information they seek when using 
the book as reference or handbook, but all text can also be read subsequently when using it as a textbook. To further support a clear structure, important definitions, checklists, and concepts are set-off in color-coded boxes:

- Facts are highlighted within blue boxes. In total, three types of these boxes can be found:

- Key Concepts (dark blue) are used to briefly emphasize the important idea behind a technical term, while

- Definitions (light blue) clearly define the term. These definitions, collected from all sections and put in alphabetical order, form the glossary.

- Pearls (light blue) are used to summarize the "take-home messages" at the end of each chapter.

- Practical information is indicated using yellow boxes. Here, four types of boxes are used:

- Our Experiences (dark yellow) narrate non-fictive situations. These boxes illustrate the presented facts and allow the reader to learn from examples. They are often concluded by a section headed "key lesson learned".

- Anecdotes (dark yellow) are told to illustrate the presented facts, to make the reader critically reflecting them, or even to provide concrete figures as they can be found in the Internet.

- Checklists (light yellow) provide sorted lists of key points that need taken into account.

- Step-by-Steps (light yellow) split algorithm and procedures into their successive components.

- Going beyond visions and concepts are emphasized by purple boxes with three different headings:

- Hypothetical Scenarios are used to tell a fictive story that is illustrating key concepts of the chapter. In addition to "Our Experience", these elements support blended learning by illustrative examples, which all are shortly discussed and concluded.

- Thought Problems are similar instruments, which are used to get the reader creatively seeking solutions of concrete problems or sample situations, such as an all of a sudden PACS malfunction reported over the phone.

- Further Readings collect references to selected textbooks and articles on the specific chapter/section/subsection.

Beside this novel approach of structuring information in a printed book, the text is also enriched with several tables and some figures, mostly in color.

Although book reviews usually summarize chapter by chapter, I found it more important to emphasize the unique concept behind the comprehensive collection of information. It is obvious that we cannot report on each chapter individually, but I have selected a chapter of each section to exemplarily report on details.
Having a strong background in medical imaging, I was particularly interested in Chapter 4, "Modalities and Data Acquisitions" (Section 1: Technology), written by J. Anthony Seibert, a well known Professor of Radiology at the University of California at Davis (UCD). He is focusing on (a) digital radiology and digital mammography, (b) digital fluoroscopy and interventional radiology, (c) computed tomography, (d) magnetic resonance imaging (MRI) and spectroscopy (MRS), (e) ultrasound, (f) nuclear medicine, and (g) other modalities, namely visible light imaging in pathology, dermatology, and ophthalmology. Within all these technologies, most relevant techniques and terms are briefly but accurately defined. Dr. Seibert also identifies network subnets for imaging devices and quality control as key concepts to have an IT-based imaging department running correctly and smoothly integrated with PACS and RIS. From the practical point of view, quality assurance (QA), and quality control (QC) are emphasized, differing whether we are operating the devices correctly, or the devices are operating correctly, respectively: "Continuous quality improvement is a key factor for acting on QA and QC findings to strive for optimal patient care". He concludes with the overarching need to consider the whole imaging system - not just the modality.

Dr. Janice Honeyman-Buck, the Editor-in-Chief of JDI, has contributed the chapter on "Customer Relations" (Section 2: Operation). Although customer satisfaction is fundamental for IT acceptance (see, for instance, ISO 9241 defining effectiveness, efficiency, and satisfaction: the comfort and acceptability of the work system to its users and other people affected by its use), customer relations usually are not a topic in textbooks for computer scientists. Introducing the chapter with an "Our Experience" report on car rentals, a situation that is familiar with all of us, Dr. Honeyman-Buck immediately is capturing the reader and guiding him trough her important messages. Ordered by radiologists, clinicians, technologists, hospital IT, hospital personal, vendors, and patients, the key issues for the IIP interacting with all this special types of customers are emphasized. Checklists provide useful tips to improve the relation, and Hypothetical Scenarios and Thought Problems are used extensively to illustrate sample problems that may occur, and how they could be handled or prevented: a customer "may sometimes be angry, [but] it is his situation and not the IIP he is attacking". Furthermore, useful tools are discussed supporting the IIP in his role as a service provider. Documentation and education can be supported by wikis, blogs, and other collaborative tools that are mostly distributed freely as open-source. PHP ticket, PHP-Nuke and Redmine are suggested. Furthermore, proactive system monitoring using digital dashboard and monitor software such as Nagios are suggested. The chapter closes with some last thoughts, repeating that IIPs have to have "a sense of humor, a sense of 
compassion, a real commitment to service, understanding of technical weaknesses in others, and respect for other people and their knowledge levels."

Chapter 21 on "PACS Readiness" (Section 3: Strategy and Vision) has been written by Steven C. Horii, Certified IIT (CIIP) and Professor of Radiology at the University of Pennsylvania. He starts with a collection of not good reasons to change from film-based radiology into the digital world, e.g., saving money or solving workflow problems. Contrarily, capacity improvement, real-time options, and tele-radiology support are worthy goals. The first step of preparation is the assessment of workload, workflow, imaging equipment, and information systems. Workflow assessment is performed using methods such as follow something, brainstorm, or hire someone. Illustrated by Our Experience reports, the "follow a film" example yields that films do not always go where you predicted, and the Picture Archiving and Communication System (PACS) must support image distributions to these diverse locations. The next question considers the system design, interfaced or integrated, the latter requiring high reliability and availability. Radiology Information System (RIS)/PACS integration is further discussed using Integrating the Healthcare Enterprise (IHE) profiles, and examples are given with advanced visualization systems, miniPACS, desktop computing, and communication networks. Personnel, however, may play a key role in PACS, and the IT manager might need accompaniment by separate PACS and/or modality managers. A chapter on change management completes the survey, emphasizing important steps such as "communicating the idea, $[\ldots]$ involve those affected, and be realistic about expectations and goals ..." Training the trainers is named as suitable concept to manage the change, and record keeping and history are also pointed crucial. For example, "if there has been a software or hardware change, and the date and nature of that change are not recorded, any effects on radiologist performance may become confounding variable in the study." Finally, Dr. Horii points out that the right external consultant can make the transition a lot smoother, and he lists the main decision points for getting external help.

After reporting on all my enthusiasm for this book, it was difficult for me to find at least some aspects of critics that are worth mention. For instance, the color concept of highlighting is somehow ambiguous and should be improved. Light and dark variants are hard to distinguish semantically, since the colors used for textbox shading are not explained, and optically, if not present on the same page. Furthermore, the Further Reading boxes sometimes are spread over the text of a chapter, and sometimes they are repetitive to the subsection Suggested Reading at the end of each chapter.

Anyway, these minor points do not lower the prominent merits of this textbook/handbook-synthesis at all. Branstetter 4th and his associated editors Rubin, Griffin, and Weiss have accomplished a considerable amount within the pages of this book and have managed to integrate a large number of complex subject areas, all of which being most relevant for applied imaging informatics. The book is unique regarding its value for praxis and application, but also provides excellent resources for theory and research with its sharp but complete definitions of terms and concepts. It should be on the desk of every IT professional in health care, directly placed between keyboard and mouse (integrated, not interfaced...). It may further be mentioned that members of the Society of Imaging Informatics in Medicine (SIIM) are granted a considerable discount on purchase.

Thomas Deserno

Aachen, Germany 